\title{
Polydiacetylene Liposomal Aequorin Bioluminescent Device for Detection of Hydrophobic Compounds
}

\section{Supporting information}

Ryoko Yamamoto, Shigehiko Takegami*, Atsuko Konishi, Hikari Horikawa, Sayumi Yonezawa,

\section{Tatsuya Kitade}

Department of Analytical Chemistry, Kyoto Pharmaceutical University, 5 Nakauchicho, Misasagi, Yamashina-ku, Kyoto 607-8414, Japan

* takegami@mb.kyoto-phu.ac.jp, Tel: +81 75595 4659, Fax: +81 755954760

Table of Contents

1 Particle size distributions of DALS and PLABD

2 Color change of PDALS at $\mathrm{pH} 9.0$ with LCN

3 Calibration curve of LCN with PLABD at $\mathrm{pH} 9.0$ 


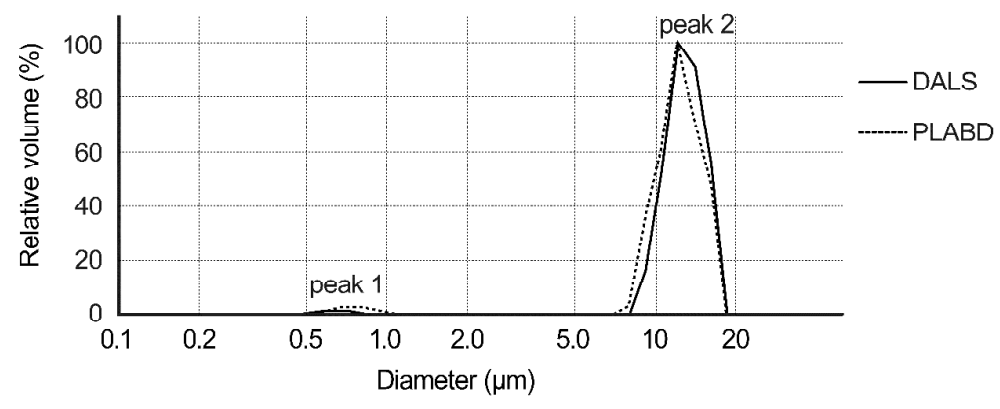

Figure S-1. Particle size distributions of DALS and PLABD determined by the DLS method. Both

DALS and PLABD showed bimodal distributions with volume-weighted distribution ratios of 2:98. 


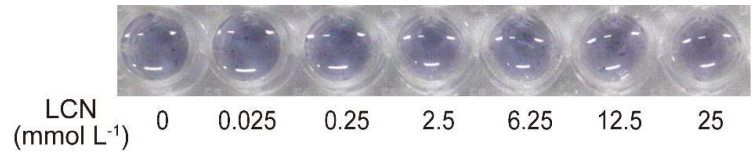

Figure S-2. The color change of PDALS suspensions at $\mathrm{pH} 9.0$ in the presence of various LCN concentrations: $0,0.025,0.25,2.5,6.25,12.5$ and $25 \mathrm{mmol} \mathrm{L}^{-1}$. 


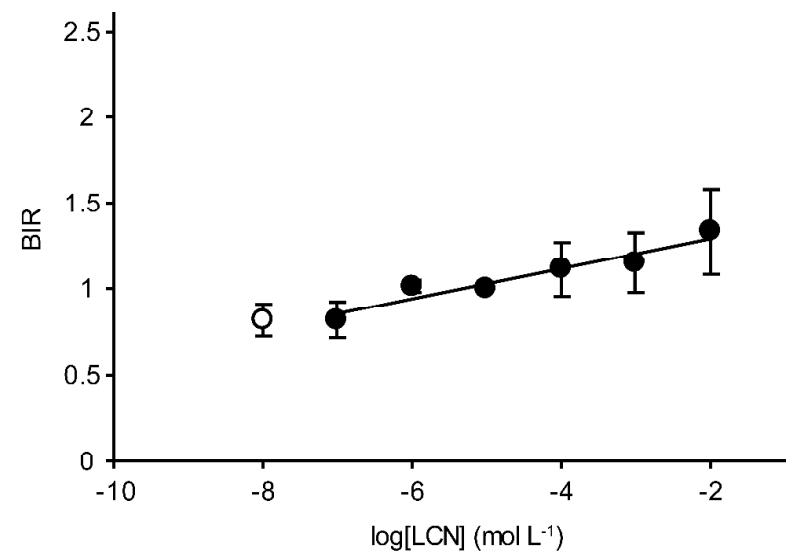

Figure S-3. The calibration curve for LCN with PLABD at $\mathrm{pH}$ 9.0. Error bars indicate the standard deviations of three replicate experiments. 